\title{
Intermittent Preventive Treatment in Infants-Adjusting Expectations and Seeing Opportunity
}

\author{
Feiko 0. ter Kuile ${ }^{1,2}$ and Richard W. Steketee ${ }^{3}$ \\ ${ }^{1}$ Liverpool School of Tropical Medicine, Liverpool, United Kingdom; ${ }^{2}$ Division of Parasitic Diseases, US Centers for Disease Control and Prevention, \\ Atlanta, Georgia; ${ }^{3}$ Malaria Control and Evaluation Partnership in Africa, PATH-Europe, Ferney-Voltaire, France
}

(See the article by Macete et al., on pages 276-85.)

Antimalarial drugs have long been used to treat acute cases of malaria, to cure infection, and to prevent disease progression, severe disease, and death. They have also been used to prevent illness (e.g., chemoprophylaxis) and to reduce transmission (e.g., mass drug administration coupled with vector control measures). With each preventive strategy, the balance of efficacy and long-term feasibility has led to progressively more focused use of the drug. That is, mass drug administration and broad use of chemoprophylaxis in a population have been reduced to targeted drug administration to fewer people and only specific groups or to shorter time intervals, to maximize benefits and to reduce costs, poor adherence, and the potential impact on drug resistance.

Among pregnant women in settings where malaria is endemic, who have long been a target population for chemoprophylaxis, studies have demonstrated that

Received 5 April 2006; accepted 5 April 2006; electronically published 30 June 2006.

Potential conflicts of interest: none reported.

Financial support: Liverpool School of Tropical Medicine (support to F.0.t.K.); US Centers for Disease Control and Prevention (support to F.O.t.K.); Program on Appropriate Technology in Health (support to R.W.S.).

Correspondence: Dr. Richard W. Steketee, MACEPA, PATHEurope, Batiment Avant Centre, 13 Chemin du Levant, 01210 Ferney-Voltaire, France (rsteketee@path.org).

The Journal of Infectious Diseases 2006; 194:269-72 (C) 2006 by the Infectious Diseases Society of America. All rights reserved.

0022-1899/2006/19403-01\$15.00 a few treatment doses of a safe and efficacious antimalarial at intervals linked to routine antenatal clinic visits could produce a substantial reduction in rates of maternal anemia, placental parasite infection, and the attendant risk of low birth weight. This proactive and presumptive (not linked to symptoms or documentation of infection) use of treatment doses at a few specific intervals, which is known as "intermittent preventive treatment (IPT) during pregnancy," has been widely adopted in many sub-Saharan African countries [1]. The concept of the timeand population-limited use of an antimalarial for a specific purpose and linked to existing routine health-care visits led to its evaluation in settings where malaria is highly endemic among infants, who have limited immunity to malaria and are at great risk of anemia and of rapid progression to severe disease and death. The demonstration by Schellenberg et al. [2] that IPT in infants (IPTi) with sulfadoxine-pyrimethamine (SP) at the time of routine immunization visits led to substantial reductions in illness and severe anemia was groundbreaking and rekindled the idea of the preventive use of antimalarials in young children. Results from an additional trial of IPTi with amodiaquine given at 3,5, and 7 months of age provided equally promising results [3]. The IPTi Consortium, a group of experienced pub- lic health scientists interested in understanding whether this approach could lead to an additional viable malaria-prevention strategy, was formed to identify the information needs and appropriate methods and studies that would clarify the value of IPTi [4].

The study reported in this issue of the Journal of Infectious Diseases by Macete et al. [5] is part of the first set of results from trials by the IPTi consortium that are centered on the safety and efficacy of IPTi with SP. These results come just 1 year after the promising results of the 2-year follow-up of the initial Tanzania study [6] and 6 months after an additional IPTi study in Ghana [7]. Although additional study results will be forthcoming, can we get a sense of where this is headed?

First, if the drug is not safe, this will all end very quickly. However, Macete et al. provide important evidence that the drug was well tolerated in infants and was not associated with adverse reactions or an adverse impact on laboratory measures and blood chemistry levels. Importantly, no adverse effects were observed on the immunogenicity of the Expanded Program of Immunization (EPI) vaccines that were administered concurrently. This is very encouraging as we await the review of these measures from the full set of studies, which will be critical in this assessment of safety. 
Table 1. Efficacy of intermittent preventive treatment in infants in Africa, from 4 completed trials.

\begin{tabular}{|c|c|c|c|c|}
\hline Study parameter & Schellenberg et al. $[2,6]$ & Chandramohan et al. [7] & Macete et al. [5] & Massaga et al. [3] \\
\hline Country & Tanzania & Ghana & Mozambique & Tanzania \\
\hline Intervention drug & SP & SP & SP & $\mathrm{AQ}$ \\
\hline Recruitment year(s) & 1999-2000 & 2000-2002 & 2002-2004 & 1999 \\
\hline Entomological inoculation rate/year & 29 & 418 & 38 & 405 \\
\hline Transmission & Perennial & Highly seasonal & $\begin{array}{l}\text { Perennial with } \\
\text { seasonal peaks }\end{array}$ & Holoendemic perennial \\
\hline Incidence rate/year of clinical malaria in placebo group & 0.36 & 1.02 & 0.43 & 2.19 \\
\hline Antimalarial drug resistance by day $14, \%(\text { drug })^{a}$ & 31 (SP) & 22 (SP) & 17 (SP) & $3(\mathrm{AQ})$ \\
\hline Use of bed nets, placebo/intervention, \% & $76 / 79^{b, c}$ & $17 / 19^{c}$ & $14 / 15^{c}$ & $30 / 32^{d}$ \\
\hline Ages at dosing, months & $2,3,9$ & $3,4,9,12$ & $3,4,9$ & $3,5,7^{e}$ \\
\hline No. of children enrolled, placebo/active & $351 / 350$ & $1242 / 1243$ & $755 / 748$ & $145 / 146$ \\
\hline Protective efficacy, \% (95\% Cl) & By 12 months & By 15 months & By 12 months & By 9 months \\
\hline Clinical malaria (all episodes) & $62.3(44.2-74.6)$ & $24.8(14.3-34.0)$ & $22.6(1.6-39.2)$ & $\begin{array}{l}64.7 \text { (42.4-77.2) (No iron) } \\
60.7 \text { (35.9-75.9) (Iron) }\end{array}$ \\
\hline Clinical malaria with high-density infection & $67.8(48.8-79.8)$ & $23.6(11.1-34.3)$ & $26.4(5.1-42.9)$ & $\begin{array}{l}72.4 \text { (52.5-83.9) (No iron) } \\
68.2 \text { (46.5-81.1) (Iron) }\end{array}$ \\
\hline All-cause hospital admissions & $30.0(8.1-46.6)$ & 12.7 ( -4.8 to 27.3$)$ & $19.0(4.0-31.0)$ & $\begin{array}{l}59.9 \text { (23.0-79.1) (No iron) } \\
52.9(12.4-74.7) \text { (Iron) }\end{array}$ \\
\hline All-cause severe anemia & $50.3(7.6-73.2)$ & $35.5(11.2-53.1)^{f}$ & $12.7(-17.3$ to 35.1$)$ & $\begin{array}{l}71.2 \text { (38.5-87.0) (Iron) } \\
74.4 \text { (43.4-88.4) (No iron) }\end{array}$ \\
\hline Rebound effect & $\begin{array}{l}\text { None: sustained effect } \\
\text { (10-24 months) }\end{array}$ & $\begin{array}{l}\text { Yes: more high-density } \\
\text { parasitemia in children } \\
16-24 \text { months old. } \\
\text { None for severe } \\
\text { anemia. }\end{array}$ & None (10-24 months) & $\begin{array}{l}\text { None (9-13 months). No } \\
\text { extended follow-up } \\
\text { data available after } 13 \\
\text { months. }\end{array}$ \\
\hline
\end{tabular}

NOTE. $\mathrm{AQ}$, amodiaquine; $\mathrm{Cl}$, confidence interval; $\mathrm{SP}$, sulfadoxine-pyrimethamine.

${ }^{a}$ Percentage of clinical and parasitological treatment failure by day 14 in symptomatic children $<5$ years old.

${ }^{b}$ Modified from Schellenberg et al. [2] by including only children with known bed-net data.

${ }^{c}$ Mostly untreated nets in the studies by Schellenberg et al. [2] and Chandramohan et al. [7]. Untreated nets only in the study by Macete et al. [5].

d Insecticide-treated nets.

e Not administered with routine vaccinations

${ }^{\dagger}$ Children hospitalized with severe anemia. 
What about efficacy? The growing spectrum of studies shows variations in efficacy (table 1). Just as we should not expect this intervention to solve all the problems of malaria in African children, we should not be surprised that the results will vary in different studies and settings. Per the IPTi Consortium plans and the standards for reporting on randomized controlled trials [4], Macete et al. report a predetermined set of outcomes. The episodes of malaria illness (with varying parasite density criteria), severe anemia, frequency of outpatient visits and hospital admissions, and other illness findings (e.g., respiratory symptoms, diarrheal illness, splenomegaly, and anemia during the short interval after dosing) were all recorded. The protective efficacy (PE) for clinical malaria with more or less strict criteria ranged from $22.2 \%$ to $26.4 \%$. The PE estimate for severe anemia was $17 \%$, but this result was not statistically significant; there was a statistically significant reduction in hospital admissions for anemia during the month after doses 1 and 2 of the antimalarial. There were significant reductions in all-cause hospital admissions (19\%), and fewer health-care facility visits for children with respiratory symptoms or diarrhea were observed, during the month after the first or second dose. The authors refrain from discussing these nonmalaria findings, but similar observations for respiratory symptoms were made in the earlier trial by Schellenberg et al. [2], which may be consistent with efficacious use of a weak antibiotic (SP) in a preventive mode. Importantly, unlike the previous study in Ghana, there was no indication of a rebound effect within the year after the completion of the third dose.

However, some of the findings were not as dramatic as those of previous reports (table 1). So is this disappointing, or does it simply demonstrate the inherent variability of malaria and its interventions? That is, the studies differ somewhat in transmission intensity; transmission seasonality; timing of dosing of the drug (starting at age 2 vs. 3 months); timing of the assessment of main end points (12 vs. 9 or 15 months); parasite resistance to the antimalarial drug; other illnesses or nutrient deficiencies affecting anemia; coverage with other malaria prevention and treatment interventions, such as iron supplementation and the use of (insecticidetreated) bed nets; and other features not measured or reported. Of interest, the SP study showing the greatest impact (in Tanzania) also had the highest coverage of bed nets. Furthermore, in the Ghana trial, the use of bed nets appeared to mitigate the effect of rebound malaria; but, in contrast to children without insecticide-treated nets, no rebound effect of high-parasite-density malaria was observed in children protected by the nets.

Nonetheless, the combined data from the 4 trials suggest that, across different investigations, with different transmission intensity and seasonality settings and even different drugs, there is substantial (albeit variable) protective efficacy of IPTi in altering the frequency of severe anemia, malaria illness, and hospital admissions. The initial enthusiasm associated with very high efficacy may be moderated with the lower protective efficacy seen in the more recent trials, but the efficacy remains and is not trivial.

Decisions about recommending the introduction of IPTi will come soon-the set of studies of the safety and efficacy of SP are expected to be formally reviewed this year. The efficacy and safety decisions derived from the individual studies and the combined analysis will offer a more complete picture for considering policy issues and next steps. The IPTi Consortium investigators will need to confront the evolving increase in antifolate resistance that could eventually preclude the use of SP. SP has a combination of features that may make it uniquely suitable for use as IPT: it is cheap, it has a relatively long halflife ( 3 days for pyrimethamine and 7 days for sulfadoxine), it is very well tolerated in young infants (unlike many other drugs), and it can be given as a single dose. The feasibility of the IPTi approach will benefit greatly from linkage with immunization programs that provide a venue to achieve high coverage with directly observed therapy at a few key time points in an infant's life. This partnership with immunization programs makes it likely that IPTi with SP will be very cost-effective even if the efficacy turns out to be more modest than was suggested in the first study. Cost-effectiveness may be somewhat less favorable, although still substantial, if some of the alternative drugs are considerably more expensive and require multiday dosing that must be done in the home. The halflife of the drug may also need to be taken into consideration when estimating how the implementation of IPTi may affect the spread of drug-resistant parasites [8]. The IPTi Consortium is addressing these issues with studies of the safety, tolerability, and efficacy of other drugs with different halflives and multiday regimens [4].

The sum of all this is the justification for having an IPTi Consortium. The public health community needs focused attention on important intervention strategies that are thoroughly probed by a set of studies that address the expected variations. The malaria-research community can benefit in many different ways from this example of a well-coordinated consortium of scientists with a comprehensive and targeted research agenda.

We have often expected too much from a single intervention. If IPTi is adopted, it will need to find its place within the scaling up of existing preventive interventions, such as insecticide-treated bed nets or indoor residual spraying. Currently, antimalarial drug use for young children in settings where malaria is endemic focuses on case management, which must rely on prompt recognition of illness and rapid action by families and health-care workers to respond to the illness. The proactive public health approach using IPTi builds on existing contacts with high-risk infants and appears to offer substantial improvement in their health. We will see, but this could be very helpful. 


\section{References}

1. World Health Organization Regional Office for Africa (WHO/AFRO). A strategic framework for malaria prevention and control during pregnancy in the African region. Brazzaville: WHO/ AFRO, 2004.

2. Schellenberg D, Menendez C, Kahigwa E, et al. Intermittent treatment for malaria and anaemia control at time of routine vaccinations in Tanzanian infants: a randomised, placebo-controlled trial. Lancet 2001;357:1471-7.

3. Massaga JJ, Kitua AY, Lemnge MM, et al. Effect of intermittent treatment with amodiaquine on anaemia and malarial fevers in infants in Tanzania: a randomised placebo-controlled trial. Lancet 2003;361:1853-60.

4. Egan A, Crawley J, Schellenberg D. Intermittent preventive treatment for malaria control in infants: moving towards evidence-based policy and public health action. Trop Med Int Health 2005; 10:815-7.

5. Macete E, Aide P, Aponte JJ, et al. Intermittent preventive treatment for malaria control administered at the time of routine vaccinations in Mozambican infants: a randomized, placebo-controlled trial. J Infect Dis 2006; 194 : 276-85 (in this issue).
6. Schellenberg D, Menendez C, Aponte JJ, et al. Intermittent preventive antimalarial treatment for Tanzanian infants: follow-up to age 2 years of a randomised, placebo-controlled trial. Lancet $2005 ; 365: 1481-3$.

7. Chandramohan D, Owusu-Agyei S, Carneiro I, et al. Cluster randomised trial of intermittent preventive treatment for malaria in infants in area of high, seasonal transmission in Ghana. BMJ 2005;331:727-33.

8. O'Meara WP, Smith DL, McKenzie FE. Potential impact of intermittent preventive treatment (IPT) on spread of drug-resistant malaria. PLoS Med 2006; 3:e141 